50(2):277-289.

11. Dror AA, Avraham KB. Hearing loss: mechanisms revealed by genetics and cell biology. Annu Rev Genet. 2009;43:411-437.

12. Kurima K, et al. Dominant and recessive deafness caused by mutations of a novel gene, TMC1, required for cochlear hair-cell function. Nat Genet. 2002;30(3):277-284.

13. Hildebrand MS, et al. Mutations in TMC1 are a common cause of DFNB7/11 hearing loss in the Iranian population. Ann Otol Rhinol Laryngol. 2010;119(12):830-835

14. Duman D, Sirmaci A, Cengiz FB, Ozdag H, Tekin M. Screening of 38 genes identifies mutations in $62 \%$ of families with nonsyndromic deafness in Tur- key. Genet Test Mol Biomarkers. 2011;15(1-2):29-33.

15 . Brownstein $Z$, et al. Targeted genomic capture and massively parallel sequencing to identify genes for hereditary hearing loss in middle eastern families. Genome Biol. 2011;12(9):R89.

16. Hrabe de Angelis MH, et al. Genome-wide, largescale production of mutant mice by ENU mutagenesis. Nat Genet. 2000;25(4):444-447.

17. Vreugde S, et al. Beethoven, a mouse model for dominant, progressive hearing loss DFNA36. Nat Genet. 2002;30(3):257-258.

18. Keresztes G, Mutai H, Heller S. TMC and EVER genes belong to a larger novel family, the TMC gene family encoding transmembrane proteins. BMC Genomics. 2003;4(1):24.
19. Labay V, Weichert RM, Makishima T, Griffith AJ. Topology of transmembrane channel-like gene 1 protein. Biochemistry. 2010;49(39):8592-8598.

20. Kurima K, Yang Y, Sorber K, Griffith AJ. Characterization of the transmembrane channel-like (TMC) gene family: functional clues from hearing loss and epidermodysplasia verruciformis. Genomics. 2003;82(3):300-308.

21. de Heer AM, et al. Progressive sensorineural hearing loss and normal vestibular function in a Dutch DFNB7/11 family with a novel mutation in TMC1. Audiol Neurootol. 2011;16(2):93-105.

22. Manor U, Kachar B. Dynamic length regulation of sensory stereocilia. Semin Cell Dev Biol. 2008; 19(6):502-510.

\title{
Slowing down with age: lung DCs do it too
}

\section{Thomas J. Braciale1,2,3 and Taeg S. Kim 1,3}

${ }^{1}$ Beirne B. Carter Center for Immunology Research, ${ }^{2}$ Department of Microbiology, and ${ }^{3}$ Department of Pathology, University of Virginia, Charlottesville, Virginia, USA.

\begin{abstract}
Decline in immune function with age has been attributed to defects or alterations in both the innate and the adaptive immune system. In this issue of the JCI, Zhao and coworkers provide evidence for a novel mechanism of immune dysfunction in aging mice. They show that migration of respiratory DCs from the site of virus replication to the draining lymph nodes in response to infection with several different respiratory viruses is markedly diminished with increasing age. The impaired DC migration was a result of increased levels of the lipid mediator prostaglandin $\mathrm{D}_{2}\left(\mathrm{PGD}_{2}\right)$ in the respiratory tract with age and could be partially reversed by blockade of $\mathrm{PGD}_{2}$ synthesis or action.
\end{abstract}

Among the many hallmarks of the aging process (in both mice and humans) is a progressive decline in immune function $(1,2)$. This is demonstrable in the diminished response of the elderly to vaccination against viral and bacterial pathogens (1). This fact compounds the more serious problem of the increased susceptibility of the elderly to infection in general and to serious and frequently life-threatening infection of the respiratory tract with respiratory viruses such as influenza A virus (IAV), respiratory syncytial virus, and severe acute respiratory syndrome coronavirus (SARS-CoV). This decline in immune function with age has been attributed to defects or alterations in both the innate and the adaptive immune system $(1,2)$. In this issue of the JCI, Zhao and coworkers provide compelling evidence for a novel mechanism of immune dysfunction in aging mice (3). They demonstrate that the migration of respiratory DCs (RDCs) from the lungs to

Conflict of interest: The authors have declared that no conflict of interest exists.

Citation for this article: J Clin Invest. 2011; 121(12):4636-4639. doi:10.1172/JCI61367. the lung draining lymph nodes (DLNs) in response to infection with several different respiratory viruses is markedly diminished with increasing age. Impaired RDC migration is mediated by the arachidonic acid (AA) metabolite prostaglandin $\mathrm{D}_{2}\left(\mathrm{PGD}_{2}\right)$, basal expression of which Zhao and coworkers show is increased in the respiratory tract with age and is further upregulated in response to infection in a pathogen-dependent fashion. They further demonstrate that blockade of either $\mathrm{PGD}_{2}$ synthesis or action partially reverses the defect in RDC migration, resulting in an enhanced adaptive immune response to infection and increased survival. These findings have interesting potential implications for both control of respiratory virus infection and vaccine efficacy in the elderly.

\section{RDC response to virus infection}

DCs are hematopoietic origin cells of the monocytic cell lineage. These cells are generally recognized to be an exclusive cell type, since they have the capacity to initiate the primary adaptive immune response (mediated by $\mathrm{T}$ and $\mathrm{B}$ cells) to foreign antigens, including infectious agents. The importance of these cells is highlighted by the fact that the 2011 Nobel Prize in Physiology or Medicine was recently awarded to Ralph Steinman for his role in the identification and characterization of DCs $(4,5)$.

DCs reside within tissues at body surfaces, such as the skin, gut, and respiratory tract mucosa. At these sites, these actively phagocytic cells act as sentinels, monitoring the site for the presence of foreign antigens, such as those derived from viruses. The tissue DCs respond to this encounter by capturing foreign antigen, processing the antigen to a form that can be recognized by $T$ cells, becoming activated, and, importantly, migrating to the DLNs from the body's surfaces. It is within the DLN that the DCs encounter T cells specific for the antigen/pathogen that they have captured and processed and, thereby, initiate the adaptive immune response (6).

Migration of activated tissue DCs from the site of infection, e.g., the lungs, to the DLN is an essential step in the induction of the adaptive immune response to the infectious agent. DC migration is chemokine regulated and, in part at least, dependent on the upregulation of expression of and signaling through the chemokine receptor CCR7, whose ligands (CCL19 and CCL21) are expressed by lymphatic endothelium draining the infected tissue (Figure 1 and ref. 7). A variety of stimuli (probably acting in concert) can stimulate tissue DC activation and, as a result, migration. These stimuli include cytokines, chemokines, and certain AA-derived metabolites, such as $\mathrm{PGE}_{2}$ and leukotriene $\mathrm{C}_{4}\left(\mathrm{LTC}_{4}\right)$ (Figure 1). Of particular relevance to the report of Zhao and coworkers is the fact that anoth- 
A
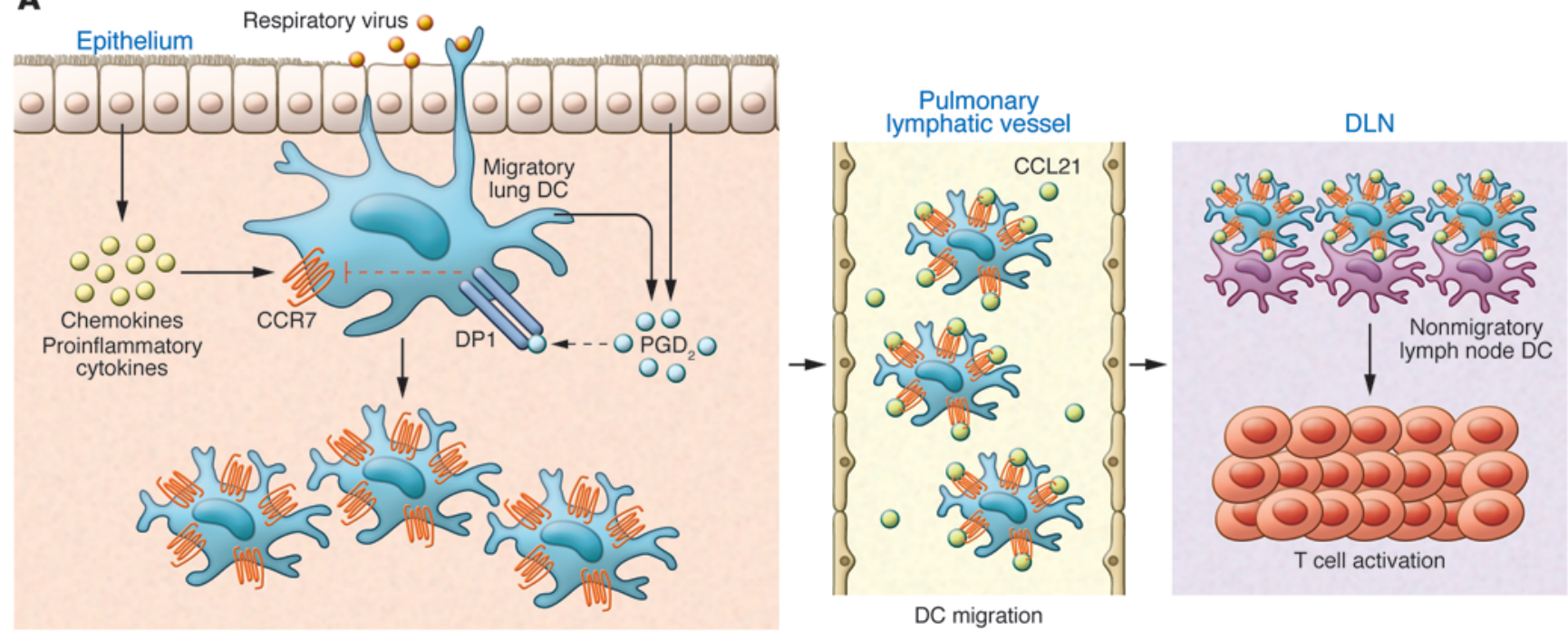

B
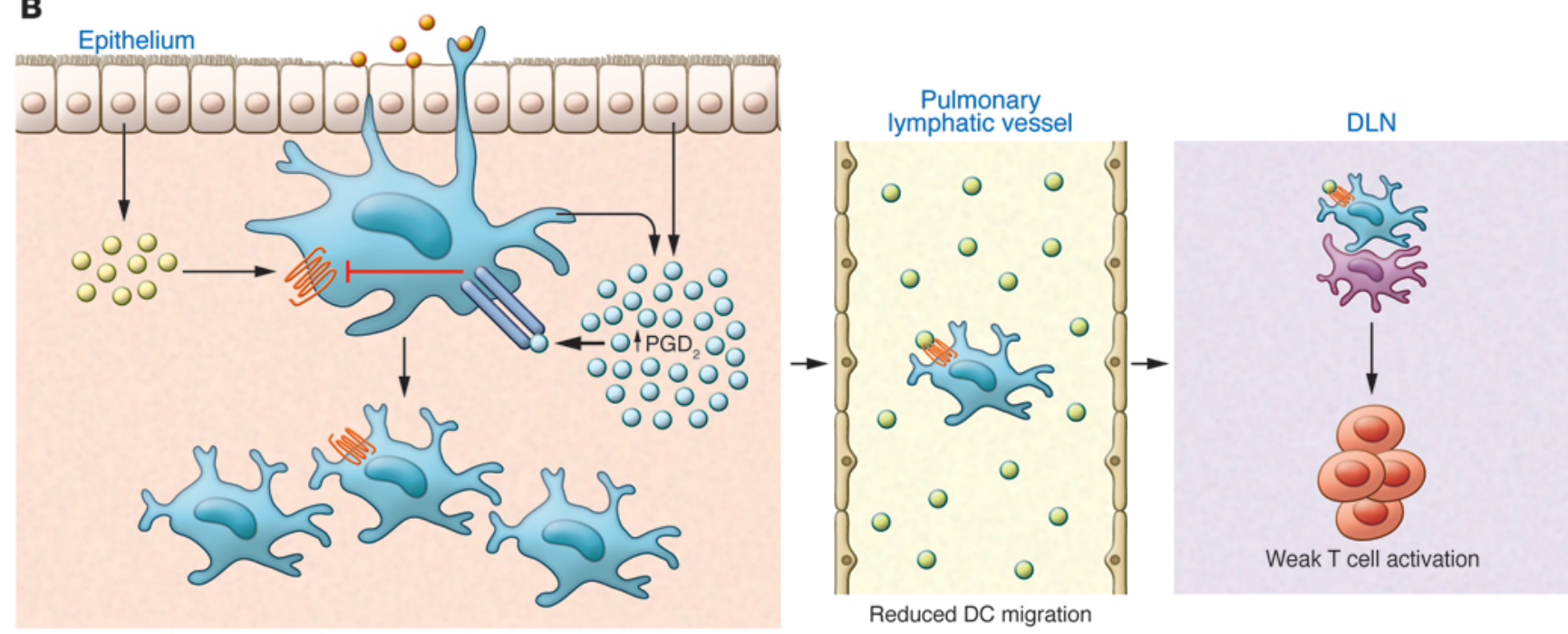

Figure 1

Impact of aging on the induction of adaptive antiviral immunity. (A) Recognition of the invading respiratory virus by innate immune receptors expressed by epithelial cells and innate immune cells, including mast cells and DCs, in the lungs leads to the production of proinflammatory cytokines (i.e., IL-1 $\beta$, IL-18, and type I IFNs), chemokines (i.e., MCP-1 and CCL2), and AA-derived metabolites (i.e., PGD 2 , PGE 2 , and LTC 4 ). Of note, basal levels of prostanoids, in particular $\mathrm{PGD}_{2}$, are increased in the lungs of aged mice. The lipid and soluble mediators in turn enhance the expression of receptors such as DP1 on DCs in the lungs. Typically in young individuals, the proinflammatory milieu in the lungs created in response to viral infection recruits and activates adjacent DCs. Activated lung DCs undergo a maturation process that includes upregulation of costimulatory ligands, antigen-presenting complexes, and, importantly, chemokine receptors (such as CCR7 and sphingosine-1-phosphate receptors [S1 $\left.\mathrm{P}_{1-5}\right]$ ). The elevated levels of chemokine receptors facilitate emigration of antigen-bearing lung DCs to the local secondary lymphoid organs draining the infected lung (DLNs), in which they participate in initiating adaptive immune response to the respiratory virus. (B) However, in the lungs of the aged, the elevated basal levels of $P \mathrm{CD}_{2}$ in the lung tissue suppress CCR7 expression on lung DCs with migratory capacity. This reduced CCR7 expression by the lung DCs impairs DC migration from the infected lung to the DLN and results in fewer viral antigen-bearing lung DCs available to activate naive virus-specific $T$ cells.

er lipid mediator derived from $\mathrm{AA}, \mathrm{PGD}_{2}$, has been previously shown to inhibit RDC migration out of the lungs $(8,9)$.

\section{AA metabolites and regulation of RDC migration}

$\mathrm{PGD}_{2}$ and $\mathrm{PGE}_{2}$ are $\mathrm{AA}$ metabolites generated through the action of 2 cyclooxygenase enzymes, the constitutively expressed COX-1 and the inducible COX-2, both of which catalyze the generation of $\mathrm{PGH}_{2}$ (Figure 2). $\mathrm{PGH}_{2}$ is in turn converted to specific biologically active prostanoids such as $\mathrm{PGD}_{2}$ by cell type-specific prostanoid synthases, e.g., $\mathrm{PGD}_{2}$ synthase (10). In the report from Zhao et al. (3), $\mathrm{PGD}_{2}$ levels were shown to become progressively elevated with age in the bronchoalveolar lavage fluid (BALF) of naive mice (i.e., prior to infection), suggesting an elevated level or at least activity of the constitutively expressed COX-1 enzyme with increasing age. Alternatively, the greater amount of $\mathrm{PGD}_{2}$ observed in the lungs of aged mice could also reflect age-associated upregulation of COX-2 expression. Senescent cells or cells derived from aged mice produce, as a by-product of the hydro- 


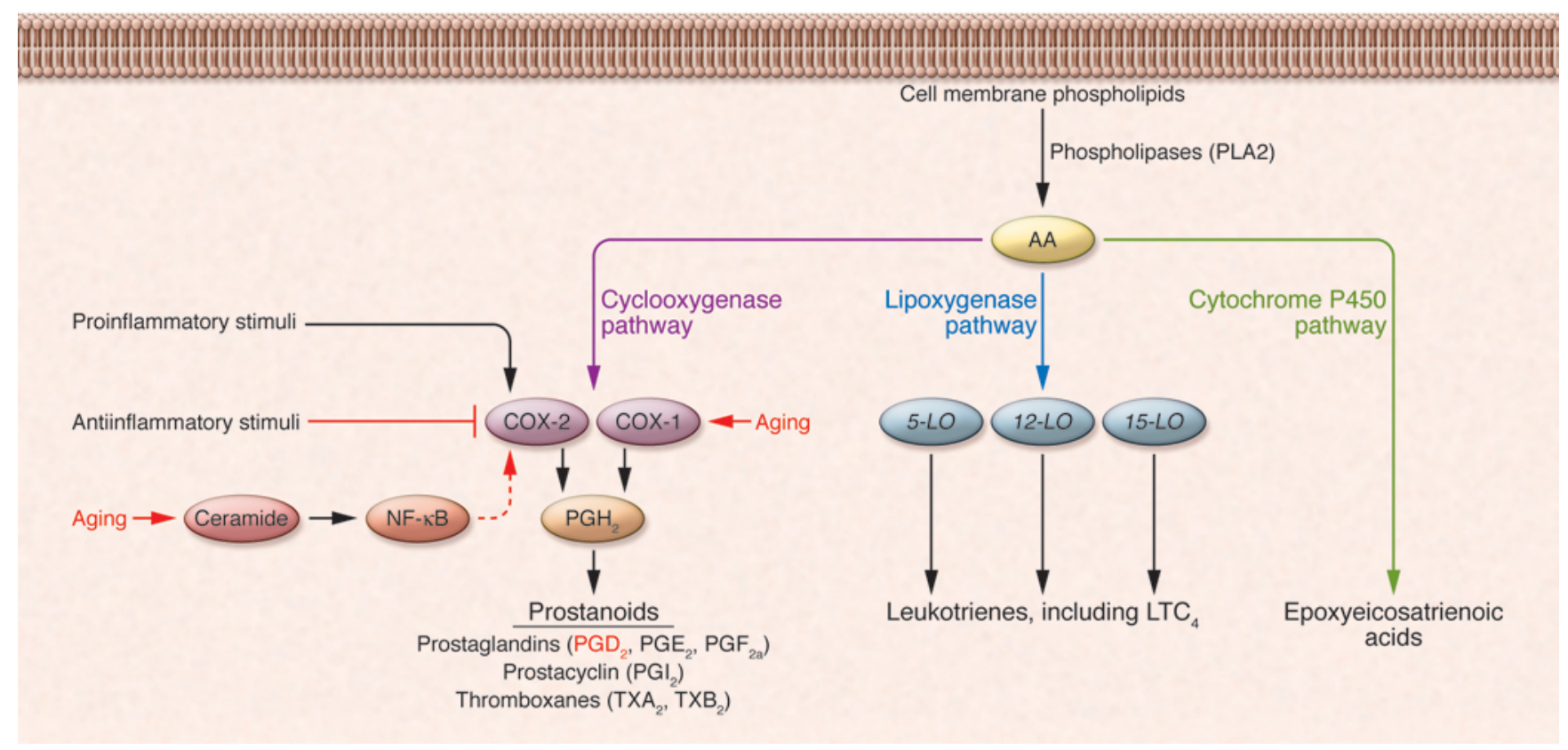

Figure 2

The pathways for eicosanoid biosynthesis. AA is released from cellular membrane phospholipids by phospholipases such as PLA2 in response to microbial infections and nonspecific stimuli, such as cytokines, hormones, and stress. Released AA can be converted to bioactive eicosanoids through the COX, lipoxygenase, or P450 epoxygenase pathways. Of interest, COX isozymes are composed of COX-1 (expression of which is constitutive) and COX-2 (expression of which is inducible). Both COX enzymes catalyze the formation of $\mathrm{PGH}_{2}$, which is converted by cellspecific prostaglandin synthases to biologically active products, collectively known as prostanoids (e.g., $P G D_{2}$ and $P G E_{2}$ ). Expression of COX-2 is induced by proinflammatory mediators and inhibited by antiinflammatory cytokines. In addition, the basal activity of COX-2 and its products, prostanoids, is observed to be elevated in both senescent cells and cells derived from aged mice. This is associated with increased amounts of ceramide, which activates NF-кB activity, leading to the transcription of COX2 mRNA. Modified from reference 10. Adapted with permission from Trends in Molecular Medicine (ref. 10).

lysis of membrane sphingomyelin, substantially more of the intracellular sphingolipid second messenger ceramide than do their younger counterparts (11). The increased levels of ceramide mediate age-associated increases in COX-2 expression and, as a consequence, prostaglandins (i.e., $\mathrm{PGD}_{2}$ and $\mathrm{PGE}_{2}$ ) levels. The elevated basal level of $\mathrm{PGD}_{2}$, detected by Zhao and coworkers in lungs of aged mice prior to infection (3), may be of biological significance, although the basal rate of RDC migration to the DLN (i.e., without an inflammatory stimulus) was not examined in these animals (Figure 1). Although it might be logistically challenging, an analysis of $\mathrm{PGD}_{2}$ levels in the human lower respiratory tract as a function of age in individuals without preexisting acute or chronic pulmonary inflammation might be warranted to complement (and substantiate) the findings of Zhao and coworkers.

Not surprisingly, Zhao et al. report that $\mathrm{PGD}_{2}$ levels increase in the BALF after respiratory virus infection (3), presumably reflecting upregulation of the inducible
COX-2 enzyme in response to proinflammatory stimuli. However, it is noteworthy that, in young mice, the effect of infection on $\mathrm{PGD}_{2}$ production was minimal, whereas, in aged mice, infection produced a greater increase in $\mathrm{PGD}_{2}$ levels. Furthermore, in the two experimental infections investigated in detail (i.e., infection with SARS-CoV and IAV), PGD $_{2}$ levels in BALF were highest in mice infected with SARS-CoV, which likewise demonstrated the greatest mortality. The authors raise the possibility that pathogen-dependent differences in $\mathrm{PGD}_{2}$ levels may reflect differences in the ability of particular viruses and specific viral gene products to regulate $\mathrm{PGD}_{2}$ levels as part of their replication strategy. The pathogen sample size studied $(n=2)$ is too small to make a definitive statement concerning this hypothesis, and factors such as virus inoculum dose, target cell types for viral replication, and virus potency in stimulating host innate responses in the respiratory tract need to be further considered. Nevertheless, these findings suggest a possible mechanism to account for the enhanced susceptibility of elderly humans to severe infection with certain respiratory pathogens.

\section{Reversal of reduced RDC migration and restoration of adaptive antiviral $\mathrm{T}$ cell immune responses by blockade of $\mathrm{PGD}_{2}$ action}

$\mathrm{PGD}_{2}$ can be produced by a variety of cell types of nonhematopoietic (i.e., epithelial cells) and hematopoietic origin, with the latter including most prominently mast cells but also tissue DCs, in response to inflammatory stimuli (ref. 10 and Figure 1). Furthermore, $\mathrm{PGD}_{2}$ can engage at least three classes of receptors: the ubiquitously expressed $\mathrm{PGD}_{2}$ receptor (DP1; also known as PTGDR), the chemokine-related receptor CRTH2 (also known as GPR44), and the nuclear receptor PPAR- $\gamma(12,13)$. In the report from Zhao and coworkers (3), the analysis of the effect of $\mathrm{PGD}_{2}$ antagonists with DP1 specificity (e.g., BW A868C) directly implicates DP1 as the receptor mediating suppression of RDC migration, interestingly, in aged but not young mice. 
Although the basis for this differential effect of DP1 antagonism on RDC migration with age remains unclear, this is in keeping with previously published observations $(8,14)$. Importantly, release of the inhibitory action of $\mathrm{PGD}_{2}$ on RDC migration by treatment with the DP1 antagonist prior to respiratory virus infection, notably SARS-CoV infection, in the aged mice resulted in the improved induction of virus-specific $\mathrm{CD}^{+} \mathrm{T}$ cells at the priming site (i.e., the DLN) and, subsequently, the infected lung, which ultimately contributed to the reduced mortality after respiratory virus infection.

\section{$\mathrm{PGD}_{2}$ controls CCR7 expression on RDCs from aged mice}

Mechanistically, Zhao et al. also provide evidence that $\mathrm{PGD}_{2}$ acts to inhibit RDC migration in aged mice by regulating (reducing) the level of expression of CCR7 on migratory RDCs in lungs as well as the frequency of CCR7 ${ }^{+}$RDCs accumulating in inflamed DLNs early in infection (ref. 3 and Figure 1). As the authors suggest, regulation of expression of the critical chemokine receptor necessary for RDC migration out of the infected lungs is likely to be one of several distinct mechanisms by which a prostanoid, such as $\mathrm{PGD}_{2}$, can act on RDC maturation and subsequent migration. In this regard, it would have been of considerable interest to evaluate, in the lungs of aged mice, the expression of other AA metabolites, notably $\mathrm{PGE}_{2}$ and $\mathrm{LTC}_{4}$, which can antagonize the effects of $\mathrm{PGD}_{2}$ $(15,16)$, and the effect of infection with the various viral pathogens on the production of these proinflammatory AA-derived lipid mediators. In this context, Zhao et al. demonstrate that administration of $\mathrm{PGE}_{2}$ to the respiratory tract of aged mice does not reverse the reduced RDC migration (3).

\section{Boosting mucosal vaccine efficacy in the elderly}

The report from $Z$ hao et al. in this issue of the JCI (3) offers a novel explanation for the increased susceptibility of the elderly to respiratory virus infections. Perhaps more importantly, these results may have therapeutic implications, as they suggest a strategy for boosting immune responses in the elderly. Although these findings do not bear on the poor response of the elderly to parenteral immunization, they do provide investigators with a new and potentially important target for investigation and manipulation, with the aim of boosting the efficacy of mucosal vaccination against respiratory viruses in the elderly. A novel mucosal vaccine formulation that included an adjuvant capable of enhancing migration of RDCs in the elderly, such as a $\mathrm{PGD}_{2}$ inhibitor, could result in improvement of the response to mucosal-administered vaccines in the elderly and lead to the use of live-attenuated virus vaccines in this population.

\section{Acknowledgments}

We thank members of the Braciale laboratory for helpful discussions. This work is supported by grants from the NIH (RO1 AI-15608, RO1 AI-37293, RO1 HL-33391, and U-19 AI-83024) to T.J. Braciale.

Address correspondence to: Thomas J. Braciale, Carter Immunology Center, University of Virginia, PO Box 801386, Charlottesville, Virginia 22908, USA. Phone: 434.924.1219; Fax: 434.924.1221; E-mail: tjb2r@virginia.edu.

1. Ongradi J, Kovesdi V. Factors that may impact on immunosenescence: an appraisal. Immun Ageing. 2010;7:7.

2. Desai A, Grolleau-Julius A, Yung R. Leukocyte function in the aging immune system. J Leukoc Biol.
2010;87(6):1001-1009.

3. Zhao J, Zhao J, Legge K, Perlman S. Age-related increases in $\mathrm{PGD}_{2}$ expression impair respiratory DC migration, resulting in diminished $\mathrm{T}$ cell responses upon respiratory virus infection in mice. J Clin Invest. 2011;121(12):4921-4930.

4. Steinman RM, Cohn ZA. Identification of a novel cell type in peripheral lymphoid organs of mice. II. Functional properties in vitro. J Exp Med. 1974;139(2):380-397.

5. Steinman RM, Lustig DS, Cohn ZA. Identification of a novel cell type in peripheral lymphoid organs of mice. 3. Functional properties in vivo.J Exp Med. 1974;139(6):1431-1445.

6. Banchereau J, Steinman RM. Dendritic cells and the control of immunity. Nature. 1998; 392(6673):245-252.

7. Tal O, et al. DC mobilization from the skin requires docking to immobilized CCL2 1 on lymphatic endothelium and intralymphatic crawling. J Exp Med. 2011;208(10):2141-2153.

8. Hammad H, de Heer HJ, Soullie T, Hoogsteden HC, Trottein F, Lambrecht BN. Prostaglandin D2 inhibits airway dendritic cell migration and function in steady state conditions by selective activation of the D prostanoid receptor 1. J Immunol. 2003;171(8):3936-3940.

9. Hammad H, et al. Activation of the D prostanoid 1 receptor suppresses asthma by modulation of lung dendritic cell function and induction of regulatory T cells. J Exp Med. 2007;204(2):357-367.

10. Harizi H, Corcuff JB, Gualde N. Arachidonic-acidderived eicosanoids: roles in biology and immunopathology. Trends Mol Med. 2008;14(10):461-469.

11. Wu D, Meydani SN. Mechanism of age-associated up-regulation in macrophage PGE2 synthesis. Brain Behav Immun. 2004;18(6):487-494.

12. Gualde N, Harizi H. Prostanoids and their receptors that modulate dendritic cell-mediated immunity. Immunol Cell Biol. 2004;82(4):353-360.

13. Sandig H, Pease JE, Sabroe I. Contrary prostaglandins: the opposing roles of PGD2 and its metabolites in leukocyte function. J Lenkoc Biol. 2007;81(2):372-382.

14. Angeli V, et al. Role of the parasite-derived prostaglandin D2 in the inhibition of epidermal Langerhans cell migration during schistosomiasis infection. J Exp Med. 2001;193(10):1135-1147.

15. Robbiani DF, Finch RA, Jager D, Muller WA, Sartorelli AC, Randolph GJ. The leukotriene C(4) transporter MRP1 regulates CCL19 (MIP-3beta, ELC)-dependent mobilization of dendritic cells to lymph nodes. Cell. 2000;103(5):757-768.

16. Scandella E, Men Y, Gillessen S, Forster R, Groettrup M. Prostaglandin E2 is a key factor for CCR7 surface expression and migration of monocyte-derived dendritic cells. Blood. 2002;100(4):1354-1361. 